\title{
A probabilistic model for strength analysis of composite double-lap single-bolt
}

\author{
joints \\ Libin Zhao ${ }^{1, *}$, Meijuan Shan ${ }^{1}$, Fengrui Liu ${ }^{1}$, Jianyu Zhang ${ }^{2, * *}$ \\ 1 School of Astronautics, Beihang University, Beijing 100191, China \\ 2 College of Aerospace Engineering, Chongqing University, Chongqing 400044, China
}

Abstract: A probabilistic model involving the randomness of basic design parameters was presented to predict the probabilistic strength of a typical composite double-lap single-bolt joint. In the probabilistic model, statistical models of the random parameters including geometrical dimensions, fiber orientation angles and material properties were constructed. In addition, a parametric failure prediction model employing a modified characteristic curve method was established. Static tensile tests of fifteen composite double-lap single-bolt joints made of T800 carbon/epoxy composites were carried out. The probabilistic failure load of the joint obtained from the proposed model is in good consistency with that from the experiments, which verifies the effectiveness and accuracy of the proposed model. Meanwhile, it shows that the proposed model is not sensitive to the probability distribution type of random variables, although the probability distribution type of random variables slightly influences the statistical parameters of the probabilistic failure load. Furthermore, a relationship between the failure probability and the tensile load is determined, and longitudinal compressive strength $X_{\mathrm{C}}$, ply thickness $t_{\text {ply }}$ and longitudinal elastic modulus $E_{11}$ of T800 unidirectional lamina are the key factors affecting the probabilistic failure load and reliability of the joint remarkably.

Keywords: Composites, Bolted joints, Probabilistic model, Modified characteristic curve method, Reliability

*Corresponding author: Libin Zhao, E-mail address: lbzhao@ buaa.edu.cn

**Corresponding author: Jianyu Zhang, E-mail address: jyzhang@ cqu.edu.cn 


\section{Introduction}

Carbon fiber reinforced plastics (CFRPs) have been increasingly utilized in aircraft structures due to their high stiffness/strength-to-weight ratio and unique designability, etc. Numerous composite bolted joints are applied to aircraft structures to connect different composite parts and transfer high loads. However, bolted joints are generally weak parts of composite structures because the drilling process causes potential damage in composites and results in remarkable stress concentration around the fastener hole. Moreover, many uncertain design parameters from the material to structure level, such as severely scattered material properties, stochastic geometric dimensions, and misaligned fiber orientation angles, etc., lead to considerable mechanical property uncertainty of bolted joints, which further imperils the safety and reliability of composite structures. Generally, to solve this problem, a large safety factor is introduced in traditional deterministic designs of composite bolted joints, which significantly diminishes the weight lose superiority of advanced composites. Thus, systematic investigations on the probabilistic design and analysis of composite bolted joints are really necessary to exert the potential of composites and achieve high reliability composite structures.

Up to now, numerous studies have been performed to quantify uncertainty in mechanical properties of composite laminates [1-2]. They focused on the probabilistic strength predictions [3-5], reliability analysis method modifications and comparisons [6-11], reliability based safety factors [12-15], reliability based design optimizations (RBDOs) [16-21] and new proposed techniques for improving computational efficiency [22-27]. Considering the specificity of hole laminates, a few researchers have endeavored to investigate the probabilistic strength of composite pin-loaded laminates with normal distribution assumptions on several design parameters, by combining a progressive damage method with statistical analysis techniques. Based on a 2D progressive damage model (PDM), Li et al. [28] employed a point estimate method and Edgeworth series to evaluate the probabilistic bearing strength of pin-loaded laminates with random material properties, and a 
maximum entropy method was recommended to replace the Edgeworth series because it can avoid the pitfall encountered in Edgeworth expansions [29]. In later research, Subset Simulation was applied to perform a more efficient probabilistic analysis of a pin-loaded laminate involving random material properties as well as fiber orientation angles [30]. Similarly, Nakayama et al. [31] combined a 2D PDM with a response surface method based Monte Carlo simulation (MCS) to predict the probabilistic strength of a pin-loaded laminate in terms of a random hole diameter and longitudinal compressive strength. Lately, they utilized two 3D PDMs to predict the probabilistic knee point strength of a pin-loaded laminate with random transverse modulus, longitudinal and transverse compressive strengths [32]. It is worthwhile noting that an effective PDM of a composite structure should be validated by a series of experiments and a progressive damage analysis consumes dramatic time and computational cost, especially for complicated composite structures. Moreover, for composite structures, uncertainties of design parameters are various, which requires more thorough discussion to accurately predict the probabilistic strength of composite structures.

The existed investigations are still limited to simple structures such as laminates and pin-loaded laminates, and it is lack of reports on the complicated bolted joints, which is a bottleneck for the safety and integrity of composite structures. In this paper, a probabilistic model was presented to predict the probabilistic strength of a composite double-lap single-bolt joint by combining MCS with a parametric failure prediction model, in which a modified characteristic curve method [33] instead of a PDM was adopted to dramatically reduce the computational cost and additional validation experiments. Basic design parameters including geometrical dimensions, fiber orientation angles and material properties were regarded as random variables and corresponding statistical models were established. Static tensile tests of fifteen joint specimens were performed to obtain the ultimate failure loads of the joint, which further provides validation for the proposed model. Furthermore, reliability and sensitivity analyses were performed to determine the failure probability of the joint as well as key influence parameters of the failure probability. 


\section{Specimens and experiments}

In order to conduct an experimental investigation on probabilistic strength of a typical composite double-lap single-bolt joint and further provide validation for the probabilistic model, fifteen specimens were manufactured and static tensile tests were performed to obtain their failure loads.

\subsection{Specimen description}

A typical composite double-lap single-bolt joint as depicted in Fig. 1 was designed and fifteen specimens were manufactured. Composite laminates of the specimens were made of T800 carbon/epoxy prepreg with the stacking sequence of $\left[\theta_{3} / \theta_{1} / \theta_{4} / \theta_{1} / \theta_{2} / \theta_{1} / \theta_{3} / \theta_{1} / \theta_{4} / \theta_{1}\right]_{\mathrm{s}}$. The nominal geometrical dimensions and fiber orientation angles are presented in Table 1, where $D$ denotes the hole diameter of the laminates and $t_{\text {ply }}$ represents the thickness of a unidirectional lamina. The length $c$ of the doublers is $75 \mathrm{~mm}$. A protruding head bolt HST12-6-7 and a high locking collar HST1078-6 both fabricated with titanium alloy were employed to join the laminates. The nominal diameter of the bolt is $4.76 \mathrm{~mm}$. An assembly tightening torque of $5.0 \mathrm{~N} \cdot \mathrm{m}$ was applied.

\subsection{Experimental procedures and results}

Static tensile tests of fifteen specimens were performed according to ASTM D 5961 [34] utilizing a testing machine Instron-8803. A tensile load was applied to the specimens by moving the lower grip holder at a loading rate of $1 \mathrm{~mm} / \mathrm{min}$ and remaining the upper grip holder still. The tensile load was continuously increased until the ultimate failure of the specimens occurred. The load-displacement $(P-\delta)$ curves of the specimens were automatically recorded by computers as represented in Fig. 2, where the peak values of the curves were deemed to be the ultimate failure loads of the specimens [34]. Table 2 further lists the ultimate failure loads of all the joints, where the mean and standard deviation of the ultimate failure loads are $21.01 \mathrm{kN}$ and $0.85 \mathrm{kN}$ respectively. The minimum and maximum values of the ultimate failure loads are $19.00 \mathrm{kN}$ and $22.34 \mathrm{kN}$ respectively.

\section{Probabilistic model}

A probabilistic model accounting for random geometrical dimensions, fiber orientation angles and 
material properties was developed to predict the probabilistic failure load of the composite double-lap single-bolt joint. In the probabilistic model, a group of statistical models was used to describe probability distribution of random design parameters. To study the effects of the probabilistic distribution type of random design parameters on the probabilistic failure load of the joint, four groups of statistical models of random design parameters were constructed by combining different probabilistic distribution types. Simultaneously, a modified characteristic curve method combined with a parametric finite element model (FEM) was employed to predict the failure load of the joint. Furthermore, a flowchart was provided to describe the implementation process of the proposed model.

\subsection{Statistical models of random design parameters}

For the double-lap single-bolt joint in this paper, the randomness of the geometrical dimensions, fiber orientation angles and material properties, which have significant influences on uncertain failure of composite bolted joints, were focused on and corresponding statistical models were established.

Table 3 lists statistics of geometrical dimensions and fiber orientation angles of composite laminates. The width $W_{1}$, end distance $L_{2}$, edge distance $W_{2}$, hole diameter $D$ as well as ply thickness $t_{\text {ply }}$ are assumed to follow a normal distribution. The means and standard deviations of each random variable are assumed according to the nominal values as well as ASTM D 5961 and $3 \sigma$ principle, respectively. Moreover, fiber orientation angles are presumed to be normal distributed with a standard deviation of 0.9 [35-37]. Concerning the fastener, only the bolt diameter is considered to be random, which is always consistent with the hole diameter of composite laminates to remain a perfect fitting state. It should be noted that all geometrical dimensions and fiber orientation angles in Table 3 are mutually independent.

As regards the material properties, the micro-level and macro-level based methods are widely utilized to obtain their statistics. The former combines a selected micro-mechanical model with 
statistics of constituent-level properties (fiber and matrix properties, fiber volume fraction, void volume fraction, et al). However, it is quite challenging to gain statistics of the constituent-level properties [38] in this method, which inevitably results in a severe limitation on its application. The latter conducts a series of coupon experiments to collect samples of the material properties and then determines their statistics by employing a specific probability distribution, such as normal, lognormal and Weibull models. Typical probability density functions (PDFs) are expressed as

$$
\begin{aligned}
& \left.f(x)\right|_{\text {Normal }}=\frac{1}{\sigma \sqrt{2 \pi}} \exp \left[-\frac{(x-\mu)^{2}}{2 \sigma^{2}}\right] \\
& \left.f(x)\right|_{\text {Lognormal }}=\frac{1}{x \sigma \sqrt{2 \pi}} \exp \left[-\frac{(\ln x-\mu)^{2}}{2 \sigma^{2}}\right] \quad x>0 \\
& \left.f(x)\right|_{\text {Weibull }}=\frac{\kappa}{\lambda}\left(\frac{x}{\lambda}\right)^{\kappa-1} \exp \left[-\left(\frac{x}{\lambda}\right)^{\kappa}\right] \quad x \geq 0
\end{aligned}
$$

For the composite material system used in this paper, the material properties are listed in Table 4. For each parameter, fifteen test values were provided by the manufacturer. To describe the statistics of the material properties, the macro-level based method is adopted by employing normal, lognormal and Weibull models. Using the parameter estimation function in software $\mathrm{Matlab}^{\circledR}$, distribution parameters of the normal, lognormal and Weibull models can be estimated based on the fifteen test values, as presented in Table 4. According to the transversely isotropic assumption, the inter-laminar elastic properties $E_{33}, G_{13}, v_{13}$ and $G_{23}$ are determined. In addition, $v_{23}$ is assumed to be equal to $v_{12}$ [39]. The equations are as follows:

$$
E_{33}=E_{22}, G_{13}=G_{12}, v_{13}=v_{12}, v_{23}=v_{12}, G_{23}=E_{22} / 2\left(1+v_{23}\right)
$$

Combining the above statistics of random variables, four groups of statistical models are established, as listed in Table 5. In all the models, geometrical dimensions and fiber orientation angles are assumed to obey a normal distribution and corresponding distribution parameters are offered in Table 3. Different combinations from normal, lognormal and Weibull distributions are assigned to elastic and strength parameters of a unidirectional lamina to explore the effects of the 
probability distribution type of random variables on the probabilistic failure load obtained from the proposed model.

\subsection{Failure prediction scheme}

Up to now, characteristic curve method is amongst the most commonly used failure prediction methods of composite bolted joints. Chang et al. [40] first developed a traditional characteristic curve method. After that, characteristic curves with different expressions were presented [41-42], as shown in Fig. 3. On consideration of the shear failure mode, Zhang et al. [33] introduced a shear characteristic length and proposed a modified characteristic curve method to attain more accurate failure predictions. The equation of the modified characteristic curve is expressed as

$$
r=R_{0}+R_{\mathrm{t}}+\left(R_{\mathrm{c}}-R_{\mathrm{t}}\right) \cos \theta+\frac{\sqrt{R_{\mathrm{s}}^{2}+R_{0}^{2}}\left[R_{\mathrm{s}}^{2}+R_{0}^{2}-\left(R_{0}+R_{\mathrm{t}}\right) \sqrt{R_{\mathrm{s}}^{2}+R_{0}^{2}}-R_{\mathrm{s}} R_{\mathrm{c}}+R_{\mathrm{s}} R_{\mathrm{t}}\right]}{R_{0} R_{\mathrm{s}}}|\sin \theta| \cos \theta
$$

where $r$ denotes the distance from points at characteristic curve to the center of hole and $R_{0}$ represents the hole radius. $R_{\mathrm{t}}, R_{\mathrm{c}}$ and $R_{\mathrm{s}}$ are the tensile, compressive and shear characteristic lengths, respectively. The angle $\theta$ is marked in a counter-clockwise direction from the symmetry axis and varies from $-90^{\circ}$ to $+90^{\circ}$. Failure mode of composite bolted joints depends on the angle $\theta$. When it satisfies $0^{\circ} \leq \theta \leq 15^{\circ}$, bearing failure occurs; $30^{\circ} \leq \theta \leq 60^{\circ}$ means shear failure and $75^{\circ} \leq \theta \leq 90^{\circ}$ represents tensile failure of the joint. For the joint specimens discussed in Section 2.1, three characteristic lengths obtained in reference [43] were utilized, which means $R_{\mathrm{t}}, R_{\mathrm{c}}$ and $R_{\mathrm{S}}$ are $0.36 \mathrm{~mm}, 1.33 \mathrm{~mm}$ and $2.94 \mathrm{~mm}$, respectively.

In the characteristic curve method, the ultimate failure of the joint can be determined once stress of a point at characteristic curve satisfies a given failure criterion. Therefore, 3D finite element technique was adopted to perform detailed stress analysis of the joint and further Yamada-Sun failure criterion [44] was employed to evaluate its ultimate failure. The equations are expressed as: 


$$
\left\{\begin{array}{l}
\left(\frac{\sigma_{11}}{X_{\mathrm{T}}}\right)^{2}=1 \quad \sigma_{11} \geq 0 \\
\left(\frac{\sigma_{11}}{X_{\mathrm{C}}}\right)^{2}+\left(\frac{\tau_{12}}{S_{12}}\right)^{2}=1 \quad \sigma_{11}<0
\end{array}\right.
$$

where $X_{\mathrm{T}}, X_{\mathrm{C}}$ and $S_{12}$ denote the longitudinal tensile strength, longitudinal compressive strength and shear strength, respectively. Consequently, an accurate failure prediction scheme based on the modified characteristic curve method was presented for the probabilistic model.

\subsection{Parametric finite element model}

As discussed above, a 3D FEM should be created to acquire detailed stress distribution of the joint. However, the basic design parameters involving geometrical dimensions, fiber orientation angles and material properties are random variables in the probabilistic model, which induces considerable difficulties for the FEM establishment. Therefore, a parametric FEM needs to be built and related techniques will be elaborated with emphasis on its differences from a deterministic FEM.

First of all, geometrical model of the joint was established with three fixed reference planes A, B and $\mathrm{C}$ as well as a local coordinate system $x$-O-y. As shown in Fig. 4, the geometrical model consists of three laminates and a fastener. Among them, three planes of the lower laminate marked by dark grey were defined as the fixed planes A, B and C. The fixed plane A is a reference for randomly varied $W_{1}$ and $W_{2}$, while the fixed plane $\mathrm{B}$ is for randomly changed $L_{2}$. It needs to note that $W_{2}$ and $L_{2}$ determine the stochastic location of the hole center, which provides reference for randomly altered hole diameter $D$. Similarly, the laminate thickness $20 * t_{\text {ply }}$ randomly varies with reference to the fixed plane $\mathrm{C}$. In addition, the random fiber orientation angles change referred to $x$-O $-y .0^{\circ}$ and $90^{\circ}$ go along the direction of $x$ and $y$ respectively. Thus, $45^{\circ}$ and $-45^{\circ}$ can be easily ascertained. All stochastic geometrical dimensions and fiber orientation angles of the lower laminate follow normal distribution. It should be noted that the middle and upper laminates are copies of the lower laminate. Therefore, their geometrical dimensions and fiber orientation angles are always accordant with the latter. With regard to the fastener, its diameter is consistent with the 
hole diameter to stay a perfect fitting state.

Moreover, a special mesh scheme was designed due to the stochastic geometrical dimensions, as depicted in Fig. 5. The mesh needs to be divided by pre-arranged seeds on the edges of the geometrical model and meanwhile the mesh size of a specific edge should be controlled with fixed element numbers. Besides, a relatively coarse mesh was chosen for non-overlap section of the laminates to cut down computational cost, while a refined radial mesh was adopted at the overlap section because of the severe stress concentration. In the inter-laminar direction, four elements were partitioned for each composite laminate. It is noteworthy that the modified characteristic curve was also utilized to divide the laminates to guarantee that the nodes will be uniformly distributed at the curve. Thus, stress of the points at the characteristic curve can be obtained directly, which avoids the possible interpolation error.

In order to simulate the actual contact states between bolt and laminate as well as laminate and laminate, contact elements were adopted for their interfaces and seven contact pairs were constructed, as shown in Fig. 5. Friction coefficient of all contact pairs was set as 0.4. Furthermore, displacement constraints in three directions $x, y$ and $z$ were applied to the section $\mathrm{A}$, and displacement constraints in directions $y$ and $z$ were applied to the section B. External load in direction $x$ was uniformly imposed on the section $\mathrm{B}$.

\subsection{Model implementation}

Based on the proposed model, a probabilistic analysis procedure was developed with the APDL of ANSYS, which contains the aforementioned statistical model of stochastic variables and parametric failure prediction model. Thus, the probabilistic failure load of composite bolted joints can be predicted with Probabilistic Design System (PDS) of ANSYS. The flowchart of the proposed model is illustrated in Fig. 6 and its main steps are described as follows:

Step 1: Define basic design parameters as input stochastic variables according to the statistical model in Section 3.1. 
Step 2: Define the ultimate failure load of the joint as an output variable.

Step 3: Select the Latin Hyper cube Sampling (LHS) technique of MCS to obtain 500 random samples of each input stochastic variable.

Step 4: Call the failure prediction module packaged from the parametric failure prediction model at cycle $i$ and assign the $i^{\text {th }}$ sample of input stochastic variables to the parametric variables.

Substep 1: Define basic design parameters including geometrical dimensions, fiber orientation angles and material properties as parametric variables with their nominal values to be initial values.

Substep 2: Establish the parametric FEM discussed in Section 3.3.

Substep 3: Calculate the failure index of nodes at the modified characteristic curve by utilizing the Yamada-Sun failure criterion.

Substep 4: If the failure index of any node equals to 1 , determine the failure load of the joint according to the tensile load; otherwise increase or decrease the tensile load and jump to Substep 3.

Step 5: If the cycle number $i$ reaches 500, acquire 500 samples of the failure load and stop the implementation; otherwise set $i=i+1$ and jump to Step 4 .

\section{Probabilistic failure load analysis}

The proposed model was utilized to predict the probabilistic failure load of the composite double-lap single-bolt joint and the experimental results in Section 2.2 were applied to validate the model. Furthermore, statistics of the probabilistic failure load were determined by employing normal, lognormal and Weibull distributions. Reliability and sensitivity analyses were performed to determine the failure probability of the joint as well as the key influence parameters of the failure probability.

\subsection{Model validation}

The proposed model was implemented in PDS of ANSYS considering four statistical models of 
basic design parameters, from which 500 samples of the failure load can be obtained. Fig. 7 represents the calculated cumulative distribution probabilities. It can be observed that the probabilistic failure load based on the four statistical models coincides well with each other, which indicates the proposed model is not sensitive to the probability distribution type of basic design parameters. Moreover, arranging the fifteen experimental values of failure load in ascending order, the cumulative probability was calculated by

$$
P_{\exp }=m /(n+1)
$$

where $m$ denote the sequence number of the value and $n$ is the number of joint specimens. The probabilistic failure load derived from the proposed model agrees well with experimental outcomes, which gives evidence that the proposed model can accurately predict the probabilistic failure load of composite bolted joints.

\subsection{Statistics of failure load}

In order to determine the statistics of the probabilistic failure load, normal, lognormal and Weibull distributions were employed to fit the 500 samples of the failure load and their cumulative distribution functions (CDFs) are as follows:

$$
\begin{aligned}
& \left.F(x)\right|_{\text {Normal }}=\frac{1}{2}\left[1+\operatorname{erf}\left(\frac{x-\mu}{\sqrt{2} \sigma}\right)\right] \\
& \left.F(x)\right|_{\text {Lognormal }}=\frac{1}{2}\left[1+\operatorname{erf}\left(\frac{\ln x-\mu}{\sqrt{2} \sigma}\right)\right] \\
& \left.F(x)\right|_{\text {Weibull }}=1-\exp \left[-\left(\frac{x}{\lambda}\right)^{\kappa}\right]
\end{aligned}
$$

where error function is $\operatorname{erf}(x)=\frac{2}{\sqrt{\pi}} \int_{0}^{x} e^{-t^{2}} d t$.

Fig. 8 further illustrates the CDFs of the probabilistic failure load fitted with Eq. (6) based on the PDS results. Clearly, for all the statistical models, the CDFs fitted by the normal distribution are always accordant with those by the lognormal distribution, which indicates that the two distributions are equivalent for describing statistics of the failure load. At the middle part, the CDFs 
fitted by the Weibull distribution are also consistent with those by the normal and lognormal distributions. However, at the lower and upper tails, the CDFs fitted by the Weibull distribution exhibit notable differences. Further, as regards to the lower and upper tails, the fitting qualities of the normal and lognormal distributions tend to be better than the Weibull distribution for statistical model I and II, whereas opposite tendency appears for statistical models III and IV. It follows that the probabilistic failure load based on statistical models I and II prefers to follow a normal/lognormal distribution, while that of statistical models III and IV inclines to obey a Weibull distribution.

Moreover, Table 6 lists the fitting parameters of the three distributions as well as the adjusted coefficient of determination $R$, which represents the goodness of the fit. All the values of $R$ are greater than 0.99, which indicates the effectiveness of the fitting results. Meanwhile, slight differences exist among the distribution parameters of the four statistical models, which shows that the probability distribution type of random variables slightly affects the statistical parameters of the probabilistic failure load of the joint obtained from the proposed model.

\subsection{Reliability analysis}

A stress-strength model was constructed to calculate the reliability of the joint, as illustrated in Fig. 9(a). The PDF of the failure load was derived from the normal distribution parameters based on the statistical model IV in Table 6. The tensile load was assumed to obey a normal distribution with $\mathrm{COV}$ of 0.05 . It needs to note that the tensile load and failure load of the joint are mutually independent. Thus, the reliability index $\beta$ can be calculated by

$$
\beta=\frac{\mu_{F}-\mu_{T}}{\sqrt{\sigma_{F}^{2}+\sigma_{T}^{2}}}
$$

where $\mu_{F}$ and $\mu_{T}$ are means of the failure load and tensile load, respectively. $\sigma_{F}$ and $\sigma_{T}$ are standard derivations of the failure load and tensile load, respectively. The failure probability $P_{\mathrm{f}}$ of the joint can be determined according to the reliability index $\beta$, 


$$
P_{f}=1-\Phi(\beta)
$$

where $\Phi(\bullet)$ is the standardized normal cumulative distribution function. Consequently, the failure probability $P_{\mathrm{f}}$ of the joint subjected to different tensile loads can be calculated from Eq. (7) and Eq. (8), as shown in Fig. 9(b). It is apparent that the failure probability of the joint monotonically increases with a higher tensile load. In addition, when the tensile load is larger than $18 \mathrm{kN}$, the uptrend is clearly strengthened.

\subsection{Sensitivity analysis}

In order to evaluate the effects of stochastic design parameters involving geometrical dimensions, fiber orientation angles and material properties on the reliability of the joint, a sensitivity analysis was conducted. A linear correlation coefficient was applied to represent the correlation between the failure load and stochastic design parameters. Fig. 10 illustrates the key influence parameters with different colors, where the left histogram denotes the values of linear correlation coefficient between the failure load and three key parameters and the right pie chart represents their relative importance. It shows that the longitudinal compressive strength $X_{\mathrm{C}}$, ply thickness $t_{\mathrm{ply}}$ and longitudinal elastic modulus $E_{11}$ are the key stochastic parameters that significantly affect the probabilistic failure load of the joint in sequence. Besides, positive correlations exist between the failure load and $X_{\mathrm{C}}$ as well as $t_{\mathrm{ply}}$, while negative correlation appears between failure load and $E_{11}$. In probabilistic strength analysis of the double-lap single-bolt joint, the final failure occurred at the compressive characteristic point or the point near the compressive characteristic point in $0^{\circ}$ plies, where $\sigma_{11}<0$ and $\tau_{12} \approx 0$. Thus, the failure was evaluated by the failure index $e=\left(\sigma_{11} / X_{\mathrm{C}}\right)^{2}+\left(\tau_{12} / S_{\mathrm{C}}\right)^{2}$ according to the Yamada-Sun failure criterion. Obviously, the increase of $X_{\mathrm{C}}$ will lead to the increase of the failure load. The increase of ply thickness $t_{\mathrm{ply}}$ results in the increase of load-carrying area, which means a larger $t_{\text {ply }}$ is followed by a larger failure load. The increase of $E_{11}$ will change the stress distribution among the plies with different fiber orientation angles, which means that the stress in $0^{\circ}$ plies will be raised and the stress in other plies will be 
reduced. Accordingly, the increase of $\sigma_{11}$ in $0^{\circ}$ plies leads to the decrease of the failure load.

It can be easily deduced from the stress-strength model that improving the reliability of the composite joint needs to increase the failure load and meanwhile decrease its deviations once statistics of the tensile load are settled. Therefore, $X_{\mathrm{C}}$ and $t_{\text {ply }}$ should be raised and $E_{11}$ should be reduced with a precondition of ensuring structure stiffness to increase the failure load of the joint and their deviations should be diminished to decrease the deviation of the failure load.

\section{Conclusions}

A probabilistic model accounting for the randomness of geometrical dimensions, fiber orientation angles and material properties was presented to predict the probabilistic failure load of a composite double-lap single-bolt joint. A parametric failure prediction model was established by employing a 3D parametric FEM and a modified characteristic curve method to predict the failure load of the joint. Four groups of statistical models of random variables were constructed with different probability distribution types, whose effects on the proposed model were discussed. Based on the APDL of the software ANSYS, a probabilistic analysis procedure was developed to implement the probabilistic failure analysis of composite bolted joints. Static tensile tests of fifteen joint specimens were performed to obtain their failure loads, which were adopted to validate the proposed model. Good consistency between the numerical and experimental results indicates that the proposed model can accurately predict the probabilistic failure load of composite bolted joints and is not sensitive to the probability distribution type of random variables.

Besides, the CDFs of the probabilistic failure load were fitted by three probability distribution types. It follows that the normal distribution and the lognormal distribution are equivalent for describing the statistics of the failure load. The Weibull distribution can obtain almost the same CDFs as the normal distribution and the lognormal distribution at the middle part, while noticeable difference appears at the lower and upper tails. Comparisons between the fitted distribution parameters show that the probability distribution type of random variables slightly influences the 
statistical parameters of the probabilistic failure load. A monotonically increasing curve of the failure probability versus the applied tensile load was presented, which is helpful for the designers to seek the quantitative relationship between the failure probability of composite joints and the applied load. Moreover, the stochastic parameters $X_{\mathrm{C}}, t_{\mathrm{ply}}$ and $E_{11}$ ranked the top three factors of significantly affecting the probabilistic failure load and reliability of the joint with the relative importance of $70 \%, 20 \%$ and $10 \%$.

\section{Acknowledgements}

The research work is supported by the National Science Foundation of China (11372020 and 11572058).

\section{References}

[1] Soares CG. Reliability of components in composite materials. Reliab Eng Syst Safe 1997; 55(2): 171-7.

[2] Chiachio M, Chiachio J, Rus G. Reliability in composites-a selective review and survey of current development. Compos Part B 2012; 43: 902-13.

[3] Yang L. Reliability of composite laminates. Mech Struct Mach 1988; 16(4): 523-36.

[4] Yang L, Ma Z. A method of reliability analysis and enumeration of significant failure modes for a composite structural system. Comput Struct 1989; 33(2): 337-44.

[5] Gurvich M, Pipes R. Probabilistic analysis of multi-step failure process of a laminated composite in bending. Compos Sci Technol 1995; 55(4): 413-21.

[6] Lin S. Reliability predictions of laminated composite plates with random system parameters. Probabilist Eng Mech 2000; 15(4): 327-38.

[7] Di Sciuva M, Lomario D. A comparison between Monte Carlo and FORMs in calculating the reliability of a composite structure. Compos Struct 2003; 59(1): 155-62.

[8] Frangopol D, Recek S. Reliability of fiber-reinforced composite laminate plates. Probabilist Eng Mech 2003; 18(2): 119-37. 
[9] Onkar A, Upadhyay C, Yadav D. Probabilistic failure of laminated composite plates using the stochastic finite element method. Compos Struct 2007; 77(1): 79-91.

[10] Lekou D, Philippidis T. Mechanical property variability in FRP laminates and its effect on failure prediction. Compos Part B 2008; 39(7-8): 1247-56.

[11] Lopes P, Gomes H, Awruch A. Reliability analysis of laminated composite structures using finite elements and neural networks. Compos Struct 2009; 92(7): 1603-13.

[12] Zhu T. A reliability-based safety factor for aircraft composite structures. Comput Struct 1993; 48(4): $745-8$.

[13] Boyer C, Béakou A, Lemaire M. Design of a composite structure to achieve a specified reliability level. Reliab Eng Syst Safe 1997; 56(3): 273-83.

[14] Richard F, Perreux D. The safety-factor calibration of laminates for long-term applications: behavior model and reliability method. Compos Sci Technol 2001; 61(14): 2087-94.

[15] Carbillet S, Richard F, Boubakar L. Reliability indicator for layered composites with strongly non-linear behavior. Compos Sci Technol 2009; 69(1): 81-7.

[16] Yang L, Ma Z. Optimum design based on reliability for a composite structural system. Comput Struct 1990; 36(5): 785-90.

[17] Miki M, Murotsu Y, Tanaka T, Shao S. Reliability-based optimization of fibrous laminated composites. Reliab Eng Syst Safe 1997; 56(3): 285-90.

[18] Ge R, Chen J, Wei J. Reliability-based design of composites under the mixed uncertainties and the optimization algorithm. Acta Mech Solida Sinica 2008; 21(1): 19-27.

[19] António C, Hoffbauer L. An approach for reliability-based robust design optimization of angle-ply composites. Compos Struct 2009; 90(1): 53-9.

[20] Young Y, Baker J, Motley M. Reliability-based design and optimization of adaptive marine structures. Compos Struct 2010; 92(2): 244-53.

[21] Gomes HM, Awruch AM, Lopes PAM. Reliability based optimization of laminated composite 
structures using genetic algorithms and Artifical Neural Networks. Struct Safe 2011; 33(3): 186-95.

[22] António CAC. A hierarchical genetic algorithm for reliability based design of geometrically non-linear composite structures. Compos Struct 2001; 54(1): 37-47.

[23] Zhang Z, Friedrich K. Artificial neural networks applied to polymer composites: a review. Compos Sci Technol 2003; 63(14): 2029-44.

[24] Wang J, Ghosn M. Linkage-shredding genetic algorithm for reliability assessment of structural systems. Struct Safe 2005; 27(1): 49-72.

[25] Kadi HE. Modeling the mechanical behavior of fiber-reinforced polymeric composite materials using artificial neural networks - a review. Compos Struct 2006; 73(1): 1-23.

[26] Au S, Ching J, Beck J. Application of subset simulation methods to reliability benchmark problems. Struct Safe 2007; 29(3): 183-93.

[27] Hurtado JE. Filtered importance sampling with support vector margin: a powerful method for structural reliability analysis. Struct Safe 2007; 29(1): 2-15.

[28] Li HS, Lu ZZ, Zhang Y. Probabilistic strength analysis of bolted joints in laminated composites using point estimate method. Compos Struct 2009; 88(2): 202-11.

[29] Li HS. Maximum entropy method for probabilistic failure load prediction of pin joints in composite laminates. Compos Struct 2013; 106: 626-34.

[30] Li HS, Xia S, Luo DM. A probabilistic analysis for pin joint failure load in composite laminates using Subset Simulation. Compos Part B 2014; 56: 780-9.

[31] Nakayama M, Uda N, Ono K. Probabilistic assessment of pin joint strength in CFRP laminates. Compos Struct 2011; 93: 2026-30.

[32] Nakayama M, Uda N, Ono $\mathrm{K}$, et al. Design-oriented strength of mechanical joints in composite laminate structures and reliability-based design factor. Compos Struct 2015; 132: $1-11$. 
[33] Zhang JY, Liu FR, Zhao LB, et al. A novel characteristic curve for failure prediction of multi-bolt composite joints. Compos Struct 2014; 108: 129-36.

[34] ASTM D 5961-13. Standard test method for bearing response of polymer matrix composite laminates. American Society for Testing of Materials, 2013.

[35] Shiao MC, Chamis CC. Probabilistic evaluation of fuselage-type composite structures. Probabilist Eng Mech 1999; 14(1-2): 179-87.

[36] Chamis CC. Probabilistic simulation of multi-scale composite behavior. Theor Appl Fract Mech 2004; 41: 51-61.

[37] Chamis CC, Abumeri GH. Probabilistic dynamic buckling of composite shell structures. Compos Part A 2005; 36(10): 1368-80.

[38] Shaw A, Sriramula S, Gosling PD, et al. A critical reliability evaluation of fibre reinforced composite materials based on probabilistic micro and macro-mechanical analysis. Compos Part B 2010; 41(6): 446-53.

[39] Feih S, Shercliff HR. Composite failure prediction of single-L joint structures under bending. Compos Part A 2005; 36(3): 381-95.

[40] Chang FK, Scott RA, Springer GS. Strength of mechanically fastened composite joints. J Compos Mater 1982; 16(6): 470-94.

[41] Xiong Y. An analytical method for failure prediction of multi-fastener composite joints. Int J Solids Struct 1996, 33(29): 4395-409.

[42] Zhang JM. Design and analysis of mechanically fastened composite joints and repairs. Eng Anal Bound Elem 2001, 25(6): 431-41.

[43] Zhang JY, Liu FR, Zhao LB, et al. Investigation on characteristic length testing methods for failure prediction of composite multi-bolt joints. J Reinf Plast Compos 2015; 34(8): 636-48.

[44] Yamada SE, Sun CT. Analysis of laminate strength and its distribution. J Compos Mater 1978; 12(3): 275-84. 


\section{Figure Captions}

Fig. 1 Schematic diagram of the specimens.

Fig. $2 P-\delta$ curves of the specimens.

Fig. 3 Sketch map of different characteristic curves.

Fig. 4 Geometrical model of the joint.

Fig. 5 Finite element model of the joint.

Fig. 6 Flowchart for predicting the probabilistic failure load of composite bolted joints.

Fig. 7 Probabilistic failure load of the joint.

Fig. 8 CDFs of the probabilistic failure load based on four statistical models: (a) Statistical model I;

(b) Statistical model II; (c) Statistical model III; (d) Statistical model IV.

Fig. 9 Reliability analysis of the joint: (a) Stress-strength model; (b) $P_{\mathrm{f}}$ vs tensile load.

Fig. 10 Sensitivity analysis of the joint. 

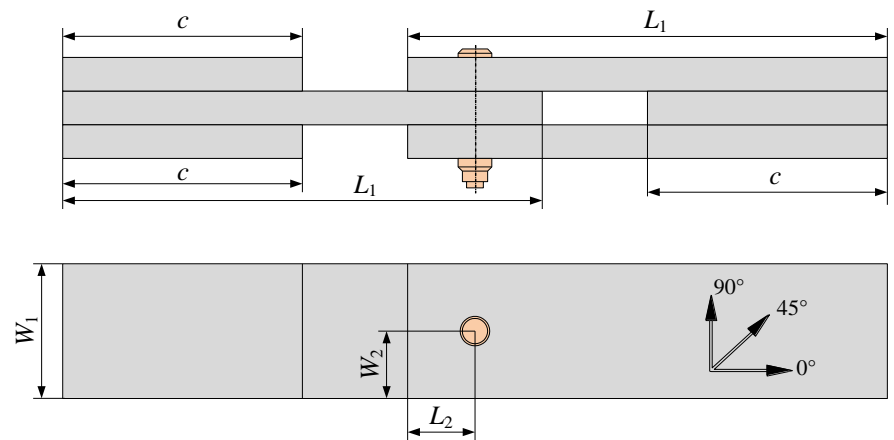

Fig. 1 Schematic diagram of the specimens

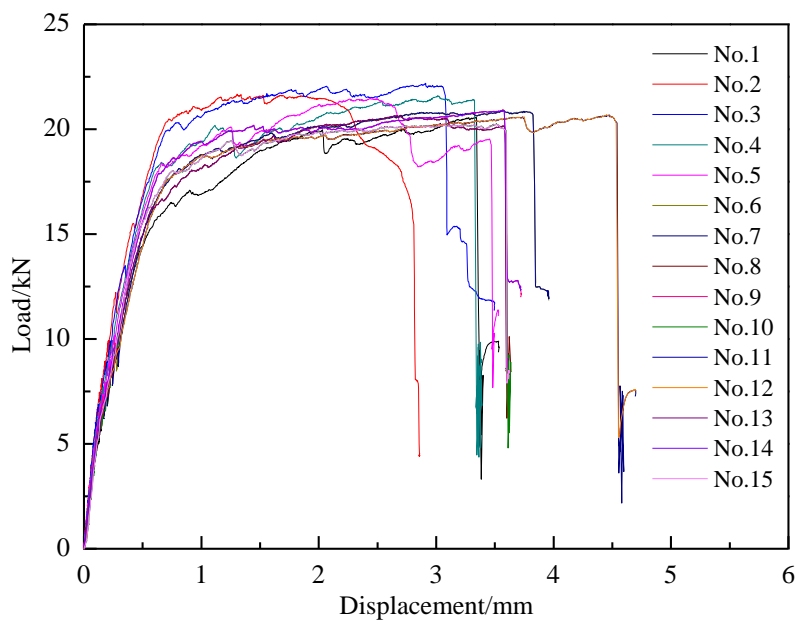

Fig. $2 P-\delta$ curves of the specimens 


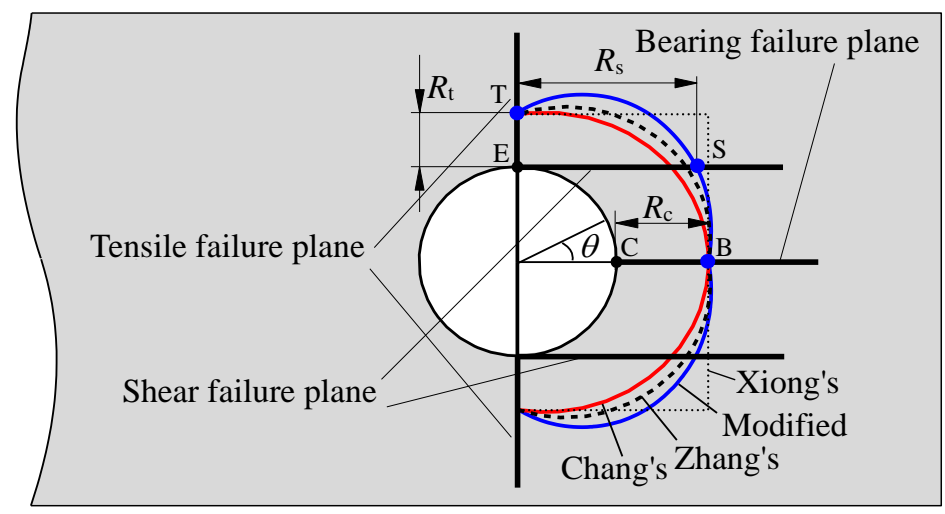

Fig. 3 Sketch map of different characteristic curves

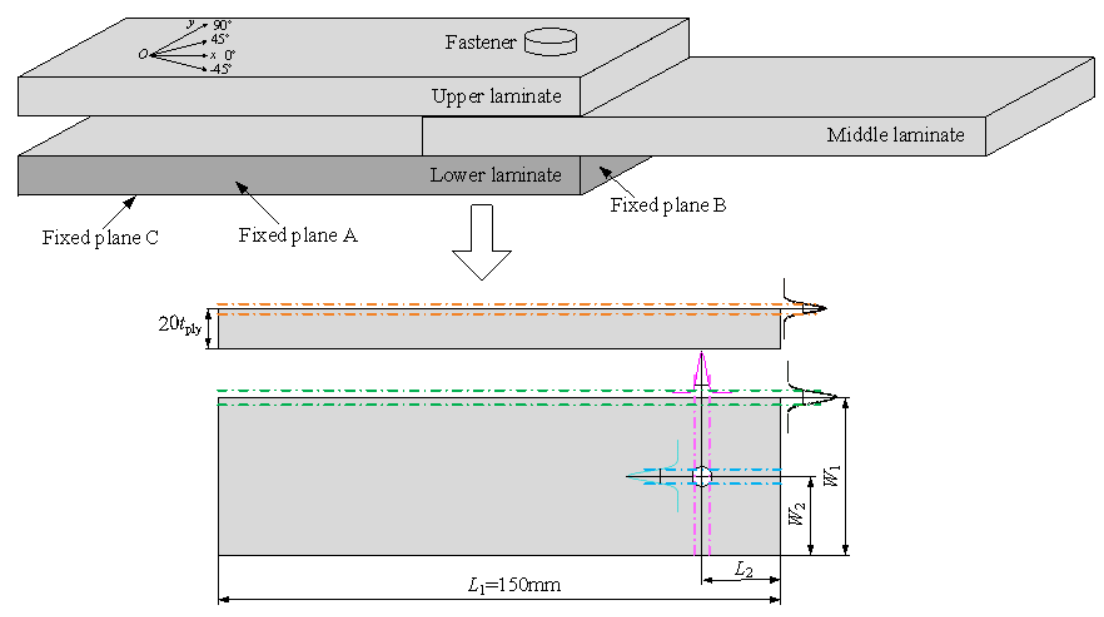

Fig. 4 Geometrical model of the joint 


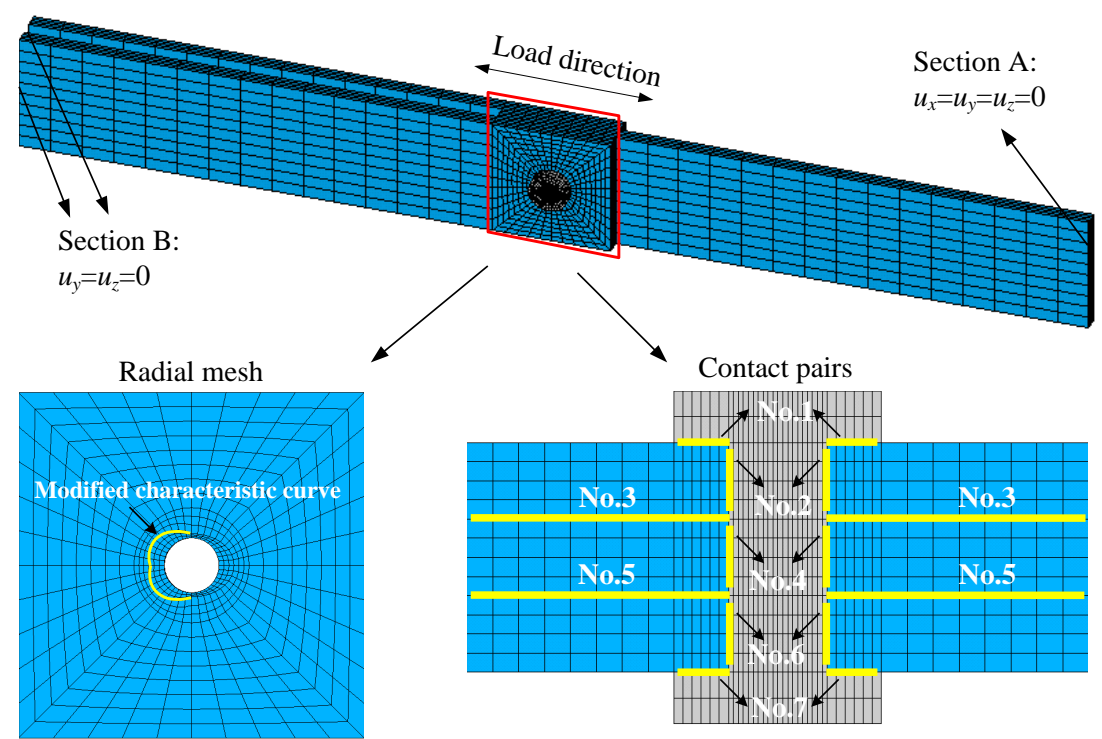

Fig. 5 Finite element model of the joint

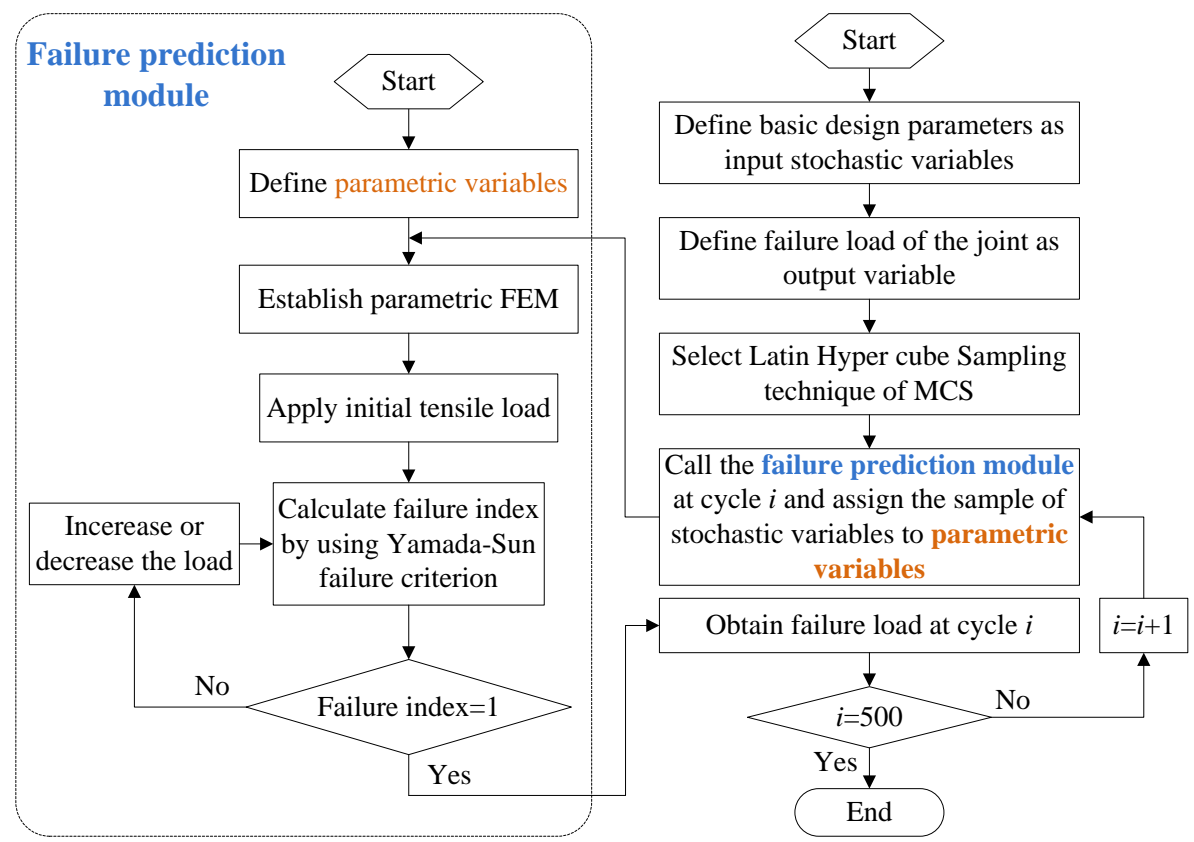

Fig. 6 Flowchart for predicting the probabilistic failure load of composite bolted joints 


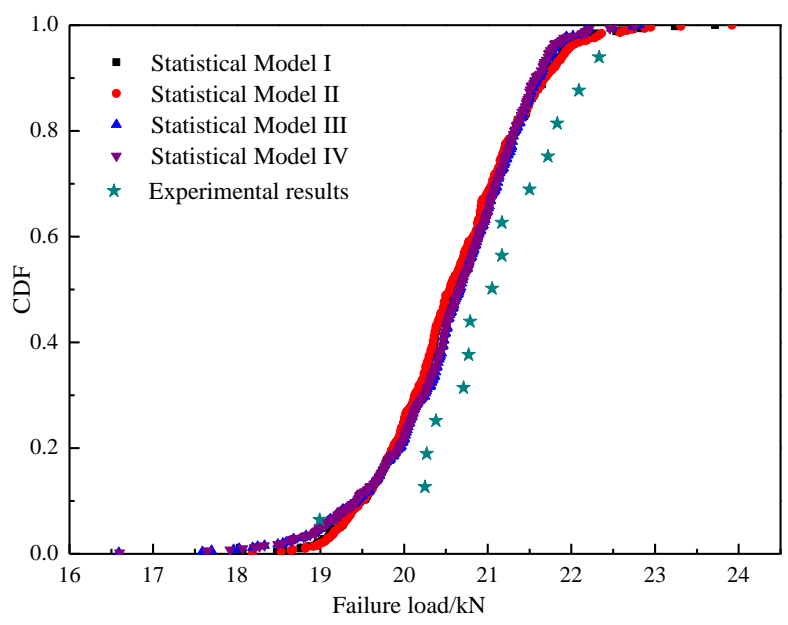

Fig. 7 Probabilistic failure load of the joint

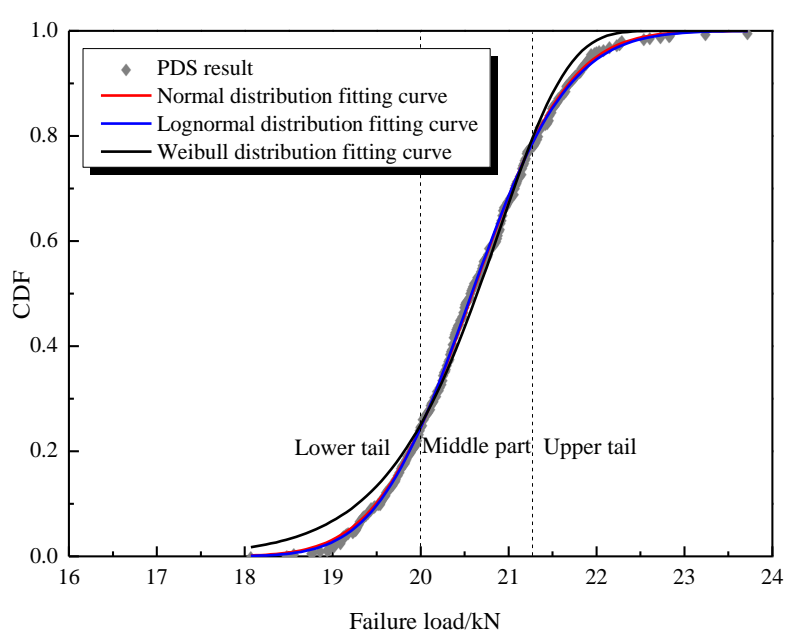

(a)

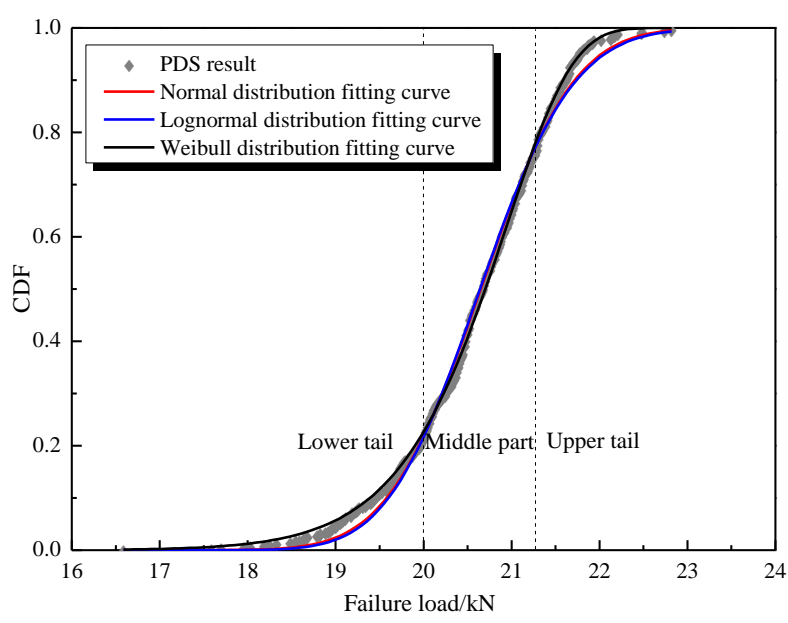

(c)

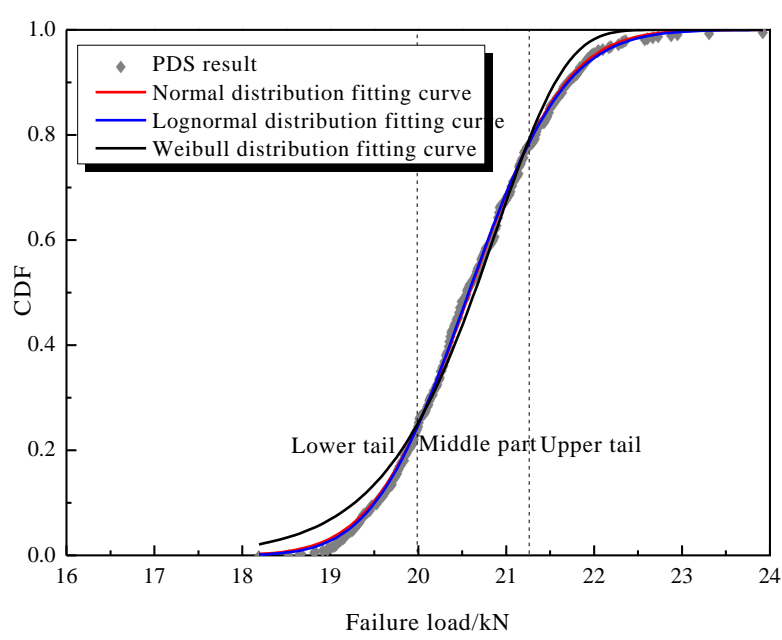

(b)

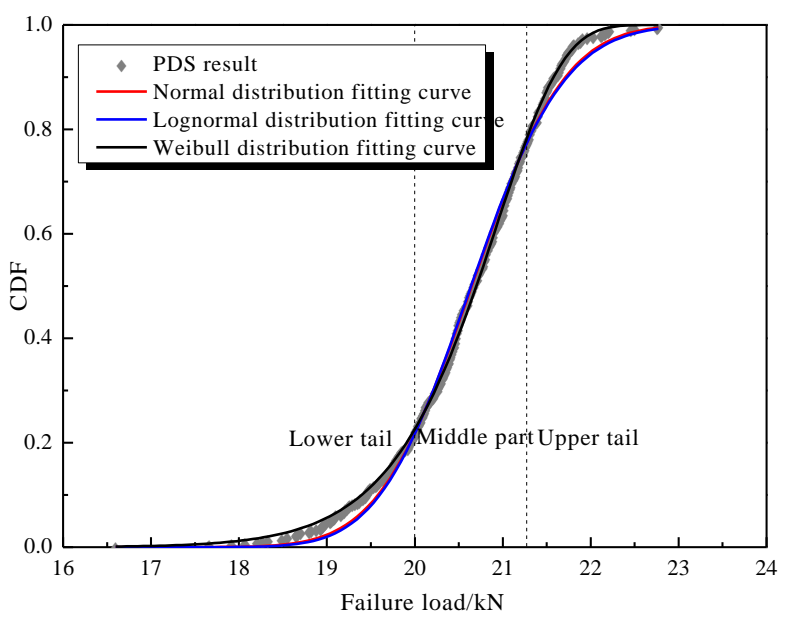

(d)

Fig. 8 CDFs of the probabilistic failure load based on four statistical models: (a) Statistical model I;

(b) Statistical model II; (c) Statistical model III; (d) Statistical model IV. 


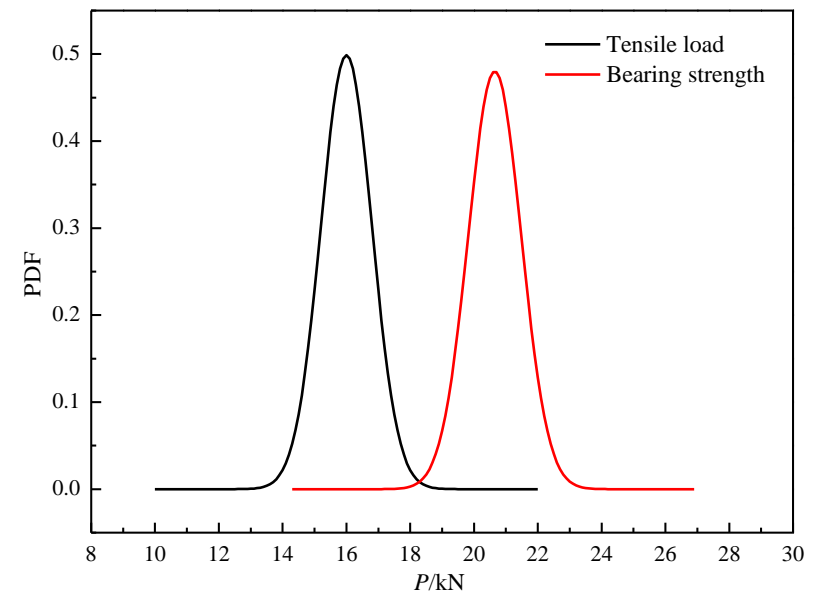

(a)

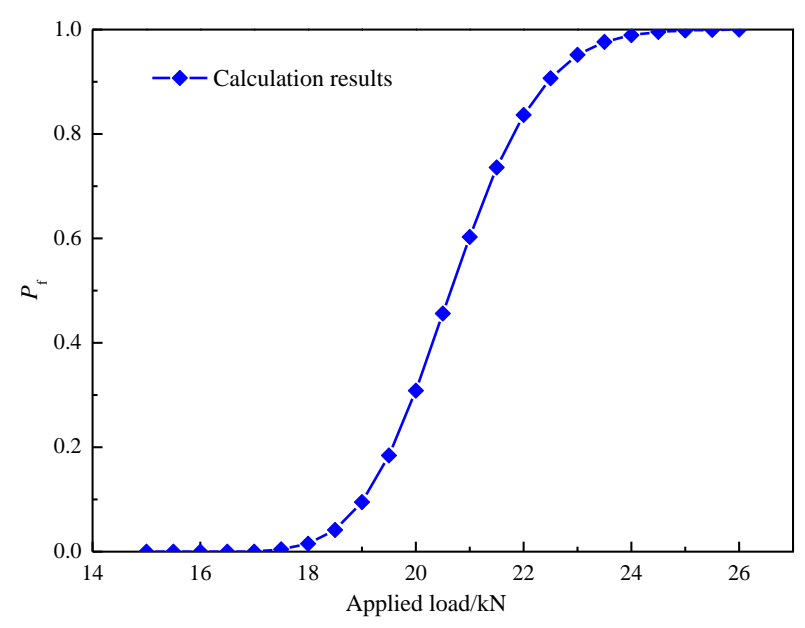

(b)

Fig. 9 Reliability analysis of the joint: (a) Stress-strength model; (b) $P_{\mathrm{f}}$ vs tensile load

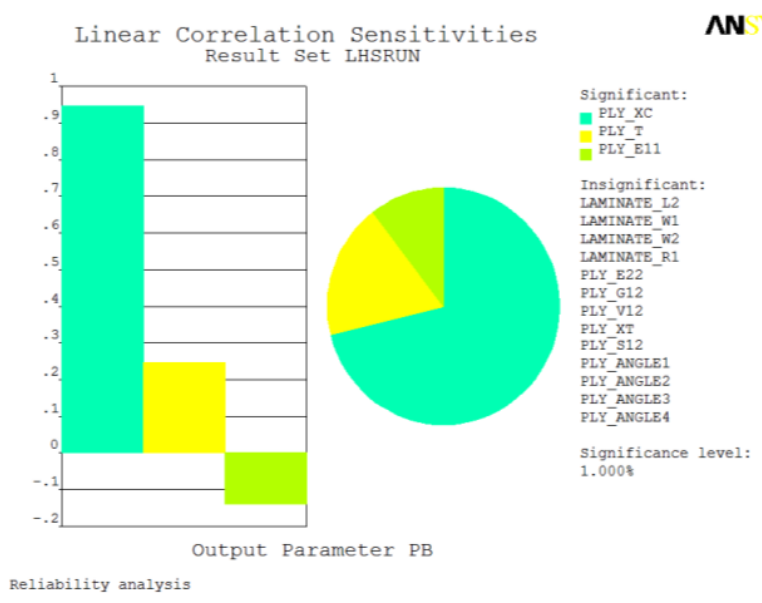

Fig. 10 Sensitivity analysis of the joint 
Table 1 Nominal geometrical dimensions and fiber orientation angles

\begin{tabular}{ccccccc}
\hline Geometrical dimensions & $L_{1}$ & $L_{2}$ & $W_{1}$ & $W_{2}$ & $D$ & $t_{\text {ply }}$ \\
Value/mm & 150 & 15 & 30 & 15 & 4.76 & 0.185 \\
\hline Fiber orientation angles & $\theta_{1}$ & $\theta_{2}$ & $\theta_{3}$ & $\theta_{4}$ & $/$ & $/$ \\
Value/ $\rho$ & 0 & 90 & 45 & -45 & $/$ & $/$ \\
\hline
\end{tabular}


Table 2 Experimental results of the ultimate failure load

\begin{tabular}{ccccccc}
\hline Specimen & Failure load/kN & Specimen & Failure load/kN & Specimen & Failure load/kN & Statistics \\
\hline No.1 & 20.72 & No.6 & 21.18 & No.11 & 19.00 & Mean $/ \mathrm{kN}$ \\
No.2 & 21.84 & No.7 & 20.80 & No.12 & 22.10 & 21.01 \\
No.3 & 22.34 & No.8 & 20.78 & No.13 & 20.39 & $S_{\mathrm{n}-1} / \mathrm{kN}$ \\
No.4 & 21.73 & No.9 & 21.06 & No.14 & 20.28 & 0.85 \\
No.5 & 21.51 & No.10 & 20.26 & No.15 & 21.18 & $/$ \\
\hline
\end{tabular}


Table 3 Statistics of geometrical dimensions and fiber orientation angles

\begin{tabular}{cccc}
\hline Random variables & $\mu$ & $\sigma$ & Distribution type \\
\hline$L_{2} / \mathrm{mm}$ & 15 & $1 / 3$ & Normal \\
$W_{1} / \mathrm{mm}$ & 30 & $1 / 3$ & Normal \\
$W_{2} / \mathrm{mm}$ & 15 & $0.5 / 3$ & Normal \\
$D / \mathrm{mm}$ & 4.76 & $0.03 / 3$ & Normal \\
$t_{\mathrm{ply}} / \mathrm{mm}$ & 0.185 & $0.012 / 3$ & Normal \\
$\theta_{1} \rho$ & 0 & 0.9 & Normal \\
$\theta_{2} \rho$ & 90 & 0.9 & Normal \\
$\theta_{3} \rho$ & 45 & 0.9 & Normal \\
$\theta_{4}{ }^{\rho}$ & -45 & 0.9 & Normal \\
\hline
\end{tabular}


Table 4 Statistics of material properties

\begin{tabular}{ccccccc}
\hline \multirow{2}{*}{ Random variables } & \multicolumn{2}{c}{ Normal distribution } & \multicolumn{2}{c}{ Lognormal distribution } & \multicolumn{2}{c}{ Weibull distribution } \\
\cline { 2 - 7 } & $\mu$ & $\sigma$ & $\mu$ & $\sigma$ & $\lambda$ & $\kappa$ \\
\hline$E_{11} / \mathrm{GPa}$ & 195 & 3.8 & 5.2735 & 0.0195 & 196.98 & 55.48 \\
$E_{22} / \mathrm{GPa}$ & 8.58 & 0.086 & 2.1491 & 0.0100 & 8.617 & 115.00 \\
$G_{12} / \mathrm{GPa}$ & 4.57 & 0.098 & -1.0898 & 0.0574 & 4.620 & 48.39 \\
$v_{12}$ & 0.34 & 0.020 & 1.5197 & 0.0212 & 0.347 & 14.52 \\
$X_{\mathrm{T}} / \mathrm{MPa}$ & 3071 & 187.4 & 8.0279 & 0.0622 & 3151.80 & 21.89 \\
$X_{\mathrm{C}} / \mathrm{MPa}$ & 1748 & 65.8 & 7.4653 & 0.0378 & 1777.74 & 32.04 \\
$S_{12} / \mathrm{MPa}$ & 143 & 3.9 & 4.9625 & 0.0276 & 144.50 & 61.28 \\
\hline
\end{tabular}


Table 5 Statistical models of basic design parameters

\begin{tabular}{ccccc}
\hline Model & Geometrical dimensions & Fiber orientation angles & Elastic parameters & Strength parameters \\
\hline No. I & Normal & Normal & Normal & Normal \\
No. II & Normal & Normal & Lognormal & Lognormal \\
No. III & Normal & Normal & Weibull & Weibull \\
No. IV & Normal & Normal & Normal & Weibull \\
\hline
\end{tabular}


Table 6 Statistics of the probabilistic failure load of the joint

\begin{tabular}{cccccccccc}
\hline \multirow{2}{*}{$\begin{array}{c}\text { Statistical } \\
\text { model }\end{array}$} & \multicolumn{3}{c}{ Normal distribution } & \multicolumn{3}{c}{ Lognormal distribution } & \multicolumn{3}{c}{ Weibull distribution } \\
\cline { 2 - 10 } & $\mu$ & $\sigma$ & $R$ & $\mu$ & $\sigma$ & $R$ & $\lambda$ & $\kappa$ & $R$ \\
\hline No. I & 20.59 & 0.856 & 0.99942 & 3.024 & 0.0416 & 0.99937 & 20.92 & 27.63 & 0.99347 \\
No. II & 20.58 & 0.856 & 0.99933 & 3.024 & 0.0416 & 0.99945 & 20.92 & 27.57 & 0.99233 \\
No. III & 20.65 & 0.834 & 0.99597 & 3.027 & 0.0404 & 0.99478 & 20.97 & 28.76 & 0.99916 \\
No. IV & 20.65 & 0.831 & 0.99601 & 3.027 & 0.0403 & 0.99451 & 20.96 & 28.95 & 0.99939 \\
\hline
\end{tabular}

